\title{
An Affordable Multichannel Potentiostat with 128 Individual Stimulation and Sensing Channels
}

\author{
Tom R. Molderez \\ dept. of Electrical Engineering (ESAT) \\ KU Leuven \\ Leuven, Belgium \\ tom.molderez@esat.kuleuven.be
}

\author{
Korneel Rabaey \\ dept. of Biotechnology \\ Ghent University \\ Ghent, Belgium \\ Korneel.Rabaey@UGent.be
}

\author{
Marian Verhelst \\ dept. of Electrical Engineering (ESAT) \\ KU Leuven \\ Leuven, Belgium \\ marian.verhelst@esat.kuleuven.be
}

\begin{abstract}
Bio)electrochemical reactions are a promising, environmentally friendly alternative for many chemical processes. These processes, however, are known to be slow in time, to be strongly dependent on the environment and to vary between different samples. This necessitates research on studying optimal operating conditions of the (bio)electrochemical cells. Yet, current experiments have to rely on slow, sequential tests. To overcome these, this work proposes a potentiostat with 128 parallel channels to speed up research experiments. The 128-channel potentiostat makes extensive use of time-sharing and is implemented with PCB technology resulting in a cost-per-channel of only $5 \$, 4 x$ lower than the state-of-the-art (SotA) and an area-per-channel of $\approx 93 \mathrm{~mm}^{2}, 5 x$ lower than the SotA. Realtime digital compensation of each individual channel is used to obtain a channel-to-channel mismatch below $1 \%$. A cyclic voltametry experiment on all channels simultaneously illustrates the low channel-to-channel mismatch. A chronoamperometry experiment with 128 different potential steps in parallel illustrates the $128 x$ experiment speedup.
\end{abstract}

Index Terms-Measurement, Microorganisms, Bioelectric phenomena, Electrochemical devices, Analog circuits

\section{INTRODUCTION}

Efficient use of resources becomes increasingly important in a circular economy. Novel electrochemical processes, catalyzed by micro-organisms, are a more environmentally friendly alternative for many classical chemical pathways. To determine the optimal electrical settings for being economically competitive, further research is required. These processes, however, are extremely slow with experiments lasting between a few days and a few weeks. Moreover, their sensitivity to the environment makes comparisons between sequential runs inaccurate. Finally, the results between similar samples in similar operation conditions vary unpredictably due to the inherent statistic variation [1].

Speeding up research experiments by reducing the experiment duration (latency) is not possible, as the slow time responses of the system are part of the subject of the studies. Yet, it is possible to achieve a speedup by increasing the amount of simultaneous experiments (throughput) by using more parallel channels. Another motivation for more parallel channels is the different response of microorganisms to static stimulation in comparison with time-varying stimulation, resulting in even more stimulation options [2]. Hence, there is a clear need to many $(>100)$ individual channels.
A potentiostat channel is used for configuring the electrical settings by both stimulation and sensing electrochemical processes. This instrument controls the voltage difference of a single redox halfreaction, the potential (stimulation), while simultaneously measuring the current through an auxiliary electrode (sensing). While currently-existing commercial potentiostats achieve top-notch performance, they only contain a limited amount $(<16)$ of parallel channels. Furthermore, the cost-per-channel ranges in the order of $1 \mathrm{k}-10 \mathrm{k} \$$ making upscaling beyond a few channels expensive.

State-of-the-art (SotA) research potentiostats have improved upon commercial potentiostats on three different aspects. First, [3] improved the portability by adding a wireless module. Second, [4] has reduced the cost-per-channel and area-perchannel to $20 \$$ and $540 \mathrm{~mm}^{2}$ respectively. Third, the amount of parallel sensing channels has been increased to 24 by [5] using PCB-technology and to 1024 by [6] using advanced, yet expensive cmos technology. Nonetheless, all these research potentiostats contain a single individual stimulation channel. In [7], sensing is done simultaneously for two current channels and six voltage channels but stimulation is not supported.

To date, the maximum number of individual parallel stimulation channels is six using PCB technology [8] and an impressive 64 using cmos technology [9]. Unfortunately, these 64 channels have only on-chip electrodes, which are very small, and require a difficult and again expensive postprocessing procedure to make them biocompatible. Moreover, a connection to external electrodes is not supported, which limits the system flexibility.

In this work, a compact, low-cost, yet accurate multichannel potentiostat architecture is presented with 128 individual stimulating and sensing channels using PCB-technology. A single potentiostat channel contains only one dedicated component, a capacitor, while the other components can be time-shared across channels. As a result, the cost-per-channel is reduced to $<5 \$, 4 \mathrm{x}$ lower than the SotA [4], and the area-per-channel is reduced to $\approx 93 \mathrm{~mm}^{2}, 5 x$ lower than the SotA [4], [8]. Despite the 128 low-cost and low-area channels, the channelto-channel mismatch is reduced to $<1 \%$.

This paper is organized as follows. First, the 128-channel potentiostat architecture is discussed in section II. Next, section III discusses the system implementation. Section IV 
TABLE I

TARgET SPECIFICATIONS For A Single CHANNEL

\begin{tabular}{l|c}
\hline \hline Specification & Target \\
\hline Potential range & $\pm 1 \mathrm{mV} \cdots \pm 5 \mathrm{~V}$ \\
Current range & $I_{\min }= \pm 1 \mathrm{nA} \ldots I_{\max }= \pm 1 \mu \mathrm{A}$ \\
Bandwidth & $>10 \mathrm{~Hz}$ \\
\hline \hline
\end{tabular}

discusses the performance of the 128-channel potentiostat and compares it to the SotA. Finally, section V concludes the paper.

\section{Architecture}

The target specifications of a single potentiostat channel are summarized in Table I. The electrical insulation properties of the discrete components and the PCB technology limit the minimum feasible current. The typical potential difference for the various (bio)electrochemical reactions determines the required voltage range. The system should support various electrochemical experiments such as (constant) chronoamperometry, (constant) chronopotentiometry and cyclic voltammetry $(\mathrm{CV})$ up to $200 \mathrm{mV} \mathrm{s}^{-1}$, resulting in a required channel bandwidth of at least $>10 \mathrm{~Hz}$. Faster dynamic experiments, like impedance spectroscopy, are not supported.

The channel architecture is discussed in a bottom-up approach. To this end, paragraph II-A discusses the low-cost and low-area architecture of a single channel. Next, Paragraph II-B discusses the use of time-sharing to allow each channel to stimulate and sense individually in parallel. Paragraph II-C discusses the scalable 128-channel topology. Finally, paragraph II-D discusses the realtime digital compensation of the current and voltage to minimize the channel-to-channel mismatch.

\section{A. A 1-channel Potentiostat}

Fig. 1a shows the topology of a classical potentiostat. An opamp in negative feedback maintains the (negative) voltage between the working electrode (WE) and the reference electrode (RE) equal to a target setpoint by controlling (stimulation) the voltage of the auxiliary electrode (CE). In parallel, a transimpedance amplifier senses the current flowing through the working electrode under this voltage setpoint (sensing). This architecture has been widely used for affordable and low-area potentiostats. Still, two opamps with several discrete components are required for each channel.

In this work, the classical potentiostat channel architecture is simplified to a single component: a capacitor (Fig 1b). Like an opamp, a capacitor is capable of maintaining the voltage of the WE, although only for a short amount of time. Because charge flows out of the capacitor, periodical replenishment is needed. Moreover, the amount of charge must be accurately controlled to maintain the potential equal to a target potential.

Both a controllable voltage source (Fig. 2a) and a controllable current pump (Fig. 2b) are capable of stimulation for periodic capacitor recharge. Current pump stimulation has three advantages in comparison with a voltage source. First, a direct relation exists between the charge current $I_{c}$ and the

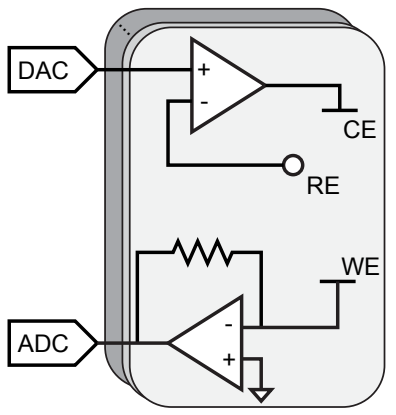

(a)

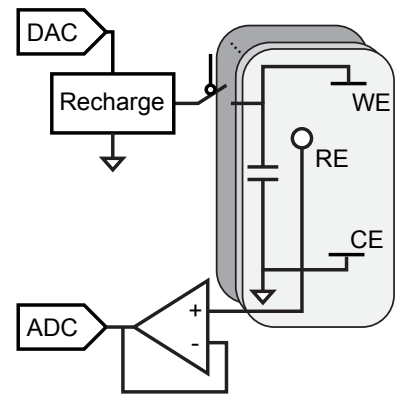

(b)
Fig. 1. Comparison between a potentiostat architecture with (a) a classical opamp-based channel and (b) a capacitor-based channel.

load current $I_{l}$ in function of the on-time $T_{o n}$ and off-time $T_{o f f}$ :

$$
I_{l}=\frac{T_{o n}}{T_{o n}+T_{o f f}} I_{c}=\frac{I_{c}}{n}
$$

where $n$ is the time-sharing ratio (or the amount of channels per recharge source). As such, there is no need for dedicated current measurement circuitry. Second, the mixedsignal sample rate should only be $1 / T_{\text {on }}$ (Fig. 2d) while for a voltage source, a much higher sample rate is required to accurately capture the recharging current wave (Fig $2 \mathrm{c}$ ). Third, the allowable voltage ripple $\Delta V_{r}$ limits $n$. For a controllable current pump, $\Delta V_{r}$ equals

$$
\Delta V_{r}<\frac{(n-1) T_{o n} I_{\max }}{C}
$$

where $C$ is the channel capacitance. Different design tradeoffs between $n, C$ and $T_{o n}$ are possible. For a controllable voltage source, due the finite on-resistance $(R \approx 100 \Omega)$ of the switch, $T_{o n}$ has a lower limit of $\tau=5 R C$. As a result, eq. 2 simplifies to:

$$
\Delta V_{r}<5(n-1) R I_{\max } .
$$

In this case, the only remaining degree of freedom is $n$.

The disadvantage of using a controllable current pump is the more complex circuitry of the source itself.

While this structure results in a channel with low cost and area (a single component), the stimulation and sensing circuitry become more complex in comparison with the classical channel architecture. This is acceptable as those can be timeshared across many channels using oversampled hardware.

\section{B. A 16-channel Potentiostat}

This design uses a timeshare ratio of $n=16$ resulting in 16 individual stimulation and sensing channels for a single current pump. The main bottleneck limiting $n$ is the available maximum multiplexer ratio of only $1 \times 16$ for discrete components with a voltage range according to Table I. To minimize the voltage ripple for this ratio, according to eq. $2, C$ should be maximized while $T_{o n}$ should be minimized. 


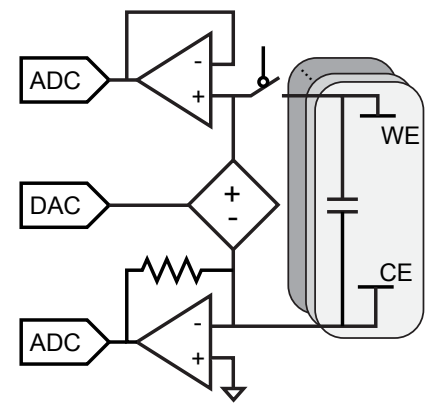

(a)

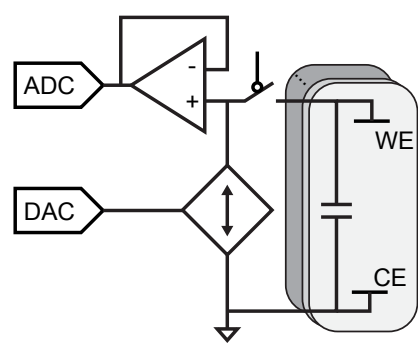

(b)

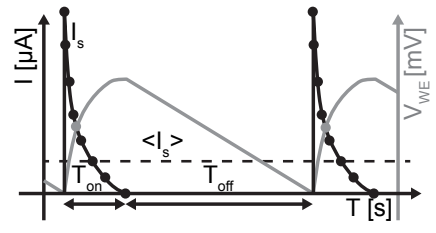

(c)

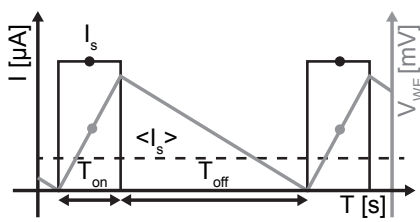

(d)
Fig. 2. Comparison between recharging with a controllable voltage source with (a) the circuitry and (c) the charge signal waves and (b) a controllable current pump with (b) the circuitry and (d) the charge signal waves. The dots indicate the discrete samples required for digitizing the signal.

The size of the channel capacitance is limited by three constraints. First, as the capacitance increases, the insulation resistance decreases, causing inaccuracies in the lower current range. Second, an increased capacitance corresponds with an increased physical size. To take advantage of the time-sharing, the area of the capacitance should be much smaller than the common current pump size. Third, a larger capacitance has a negative influence on the bandwidth. Based on these constraints, the channel capacitance is limited to $0.33 \mu \mathrm{F}$.

The minimal $T_{o n}$ is determined by the speed of the controllable current pump. During switching, a small transient charge flows because of the finite rise-time $\left(T_{r} \approx 4 \mu \mathrm{s}\right)$ and fall-time ( $T_{f} \approx 4 \mu \mathrm{s}$ ) of the current pump [8]. This unknown, transient charge must be much lower than the supplying charge to not affect the accuracy. Or equivalently, for a relative accuracy $r$, $T_{\text {on }}>\left(T_{r}+T_{f}\right) r^{-1}$.

Combining previous constraints, for $r=1 \%$, results in a theoretical maximum voltage ripple of $\Delta V=20 \mathrm{mV}$ which is high but still acceptable.

\section{A 128-channel Potentiostat}

The complete 128-channel potentiostat consists of eight 16-channel groups, all driven by a single 8-channel DAC. A single 16-channel ADC is used to measure the voltage $V_{W E}$ successively of each group (8 channels) as well as the voltage $V_{R E}$ of 8 different RE electrodes (Fig. 3). This allows to calculate the potential of each channel digitally by $E_{W E}=V_{W E}-V_{R E}$.

Each channel contains a dedicated digital LQI controller to determine the successive periodic recharge or discharge current for each channel capacitor to maintain the potential equal to

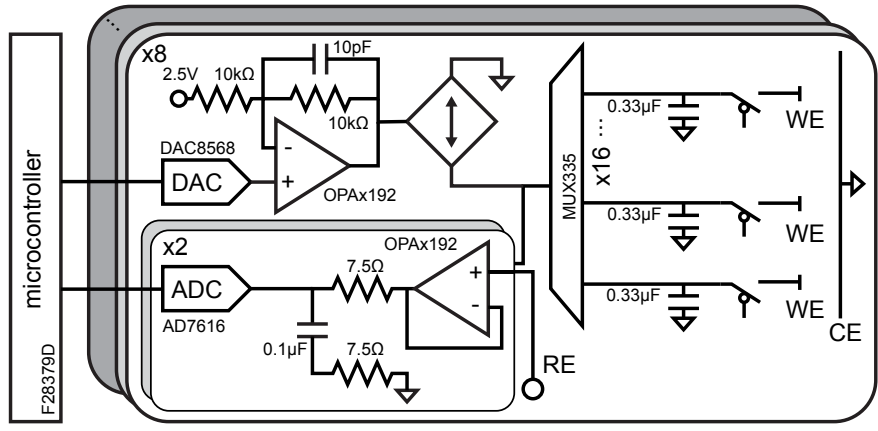

Fig. 3. Hardware architecture of the 128-channel potentiostat with 8 groups of 16 timeshared channels all driven by a single microcontroller.

the target setpoint. All LQI controllers run simultaneously in round-robin on a single processor of a microcontroller connected to the multi-channel potentiostat PCB..

\section{Realtime Digital Compensation}

Realtime digital compensation of the stimulated currents and of the sensed voltages is done to minimize the channelto-channel mismatch.

For the current stimulation, a static quadratic least-square model is used to take into account nonidealities of $I_{\text {out }}[i]$, the output of the controllable current source, and possible disturbances of the load voltage $V_{\text {out }}[i]$ :

$$
I_{\text {out }}[i]=A[i]+B[i] I_{\text {in }}[i]+C[i] V_{\text {out }}[i]+D[i] I_{\text {in }}[i] V_{\text {out }}[i]
$$

where $i$ is the channel index ranging from 1 to $128, I_{i n}[i]$ the input of the channel current source and $A[i], B[i], \ldots, D[i]$ are fitting parameters. The inverse of eq. 4 ,

$$
I_{\text {in }}[i]=\frac{I_{\text {out }}[i]-A[i]-C[i] V_{\text {out }}[i]}{B[i]+D[i] V_{\text {out }}[i]},
$$

is used to calculate the compensated input current to have the output current equal to the target current. The fitting parameters are empirically determined for each channel using an automated calibration measurement setup. For each channel, $V_{\text {out }}[i]$ is swept between $-8,-6, \ldots, 8 \mathrm{~V}$ and $I_{\text {in }}[i]$ is swept between $(0, \pm 2, \pm 4, \ldots, \pm 10) *(1,10,100 \mathrm{nA})$. This calibration experiment is run twice: first without compensation to fit the parameters and second with compensation by eq. 5 to verify the accuracy of the compensated output.

For the voltage sensing, a static linear first-order model is used:

$$
V_{\text {out }}[i]=E[i]+F[i] V_{\text {in }}[i]
$$

where $V_{i n}[i]$ is the actual channel voltage, $V_{\text {out }}[i]$ the sensed channel output voltage and $E[i]$ and $F[i]$ are fitting parameters. The inverse of eq. 6 ,

$$
V_{\text {in }}[i]=\frac{V_{\text {out }}[i]-E[i]}{F[i]},
$$

is used to calculate the actual input voltage $V_{i n}$ from the measured voltage $V_{\text {out }}$. 
These fitting parameters are again empirically determined for each channel. In this case, $V_{i n}[i]$ of each channel is swept between $-8,-6, \ldots, 8 \mathrm{~V}$, again first for fitting and second for verification of the compensation by eq. 7 .

\section{IMPLEMENTATION}

The 128-channel potentiostat is implemented with PCB technology and off-the-shelf components. The embedded digital control of each channel is implemented in $\mathrm{C}$ on a dualcore (only one core used) commercial microcontroller (Delfino F28379D, TI).

The analog hardware stimulates all working electrodes (paragraph III-A) and senses the corresponding potential response (paragraph III-B). Paragraph III-C discusses the PCB.

\section{A. The Stimulation Hardware}

The stimulation hardware (Fig. 3) is implemented with an eight-channel, 16bit DAC (DAC8586, TI), connected to eight bipolar current pumps. Each current pump is implemented according to [8] but with a different current scaling resistor value $(35.7 \mathrm{k} \Omega$ instead of $511 \Omega)$ to obtain a more sensitive current range $( \pm 100 \mathrm{pA} \cdots \pm 3.3 \mu \mathrm{A})$. Each current pump is then connected to the channel capacitors using a $1 \times 16$ analog multiplexer (MUX506, TI). This multiplexer is used because of its superb switch insulation resistance and minimal switching charge injection. The channel capacitors are metallized polyester capacitors (MKS 2, WIMA) with an excellent tradeoff between capacitance $(0.33 \mu \mathrm{F})$, area $\left(2.5 x 7.2 \mathrm{~mm}^{2}\right)$ and insulation resistance $(>10 \mathrm{G} \Omega$ ). Finally, a digital controllable switch (MAX335, Maxim Integrated) is added to enable or disable each individual channel. An additional I2C to 16 GPIO ports expander (TCA9555, TI) is added to control all those switches separately.

\section{B. The Sensing Hardware}

The sensing hardware (Fig. 3) consists of 16 equal paths of which eight are used for sensing the WE voltage of each group and eight are used for sensing the RE voltage of eight individual reference electrodes. Each of those paths contains a pseudo-differential first-order analog anti-aliasing filter with pole frequency of $100 \mathrm{kHz}$. All these filter outputs are then connected to a 16-channel, 2Ms, 16bit, SAR ADC (AD7616, Analog Devices). The input voltage range of each channel of the ADC is programmed to $\pm 10 \mathrm{~V}$.

\section{The 128-channel PCB}

Fig. 4 shows the resulting PCB. The PCB contains 8 layers: a top routing layer, a ground plane, a first inner routing layer, a ground plane, a power plane, a second inner routing layer, a power plane and a bottom routing plane. The total board area is $1.6 \times 10^{4} \mathrm{~mm}^{2}$ of which $1.2 \times 10^{4} \mathrm{~mm}^{2}$ is functional area and $4 \times 10^{3} \mathrm{~mm}^{2}$ is support area (power supply, connector to the microcontroller and connector to the external working electrodes). This results in an area-per-channel of $93 \mathrm{~mm}^{2}$. The total component cost (for 10 production units) is about $600 \$(85 \$$ for the pcb, $475 \$$ for the electronic components and

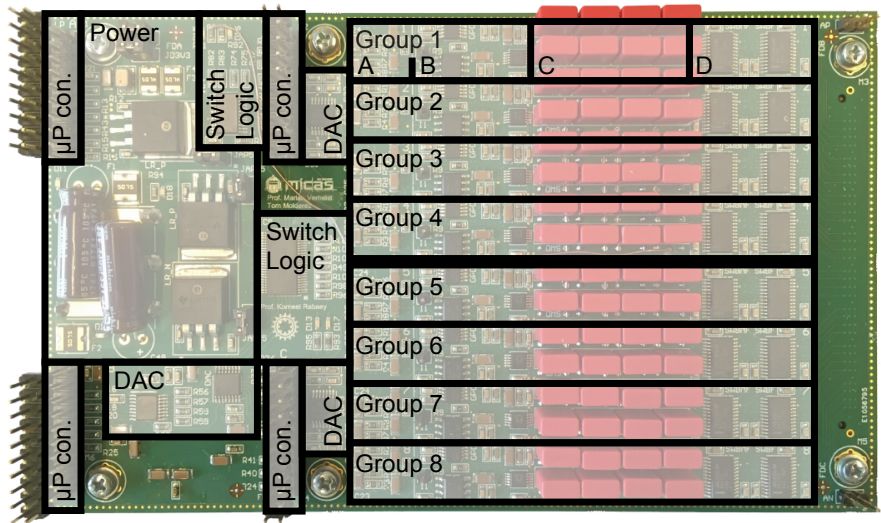

(a)

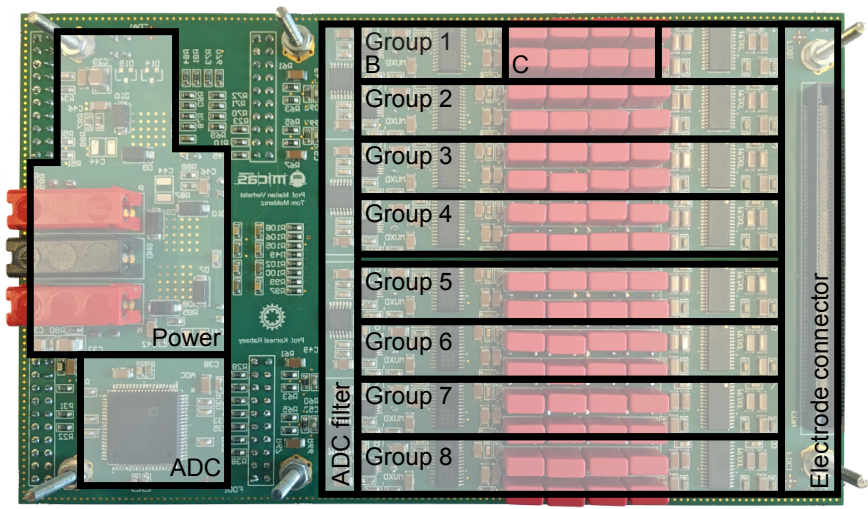

(b)

Fig. 4. PCB of the 128-channel potentiostat with (a) the front view and (b) the rear view. Each group of 16 channels contains an anti-aliasing filter (A), a current pump (B), the channel capacitors (C) and the channel switches (D).

$40 \$$ for the microcontroller) resulting in a cost-per-channel of $4.7 \$$.

\section{RESUlTS AND DISCUSSION}

The electrical performance for each channel is verified and described in paragraph IV-A. Next, the potentiostat functionality is verified with a benchmark electrochemical experiment in IV-B. Finally, paragraph IV-C compares the performance of the 128-channel potentiostat with the SotA.

\section{A. Electrical Performance}

The DC performance (accuracy and channel-to-channel mismatch) of each channel is assessed, first for the current stimulation, next for the voltage sensing.

Ideally, the output current of the current pump is only dependent on the controllable digital input (with an offset error and gain error). However, the output current is in practice also affected by the output load voltage. Fig. 5a and Fig. 5b show the impact of different load voltages on the output current of a particular channel without compensation. Especially in the lower current range $(<10 \mathrm{nA})$ is the relative current error $(10 \%-100 \%)$ strongly influenced by the load voltage, except in the region where by coincidence, the offset error equals the reverse of the gain error. Fig. 5c and Fig. 5d show the 


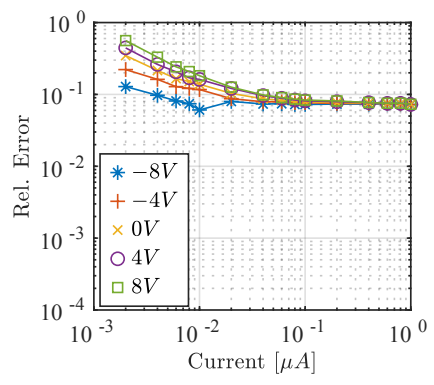

(a)

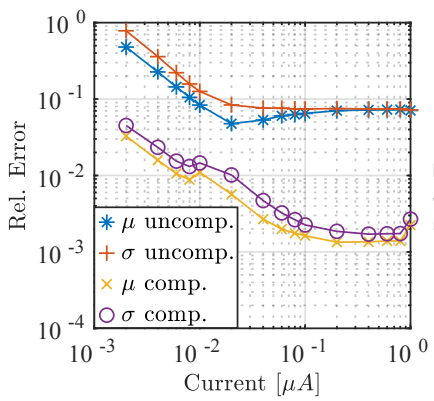

(c)

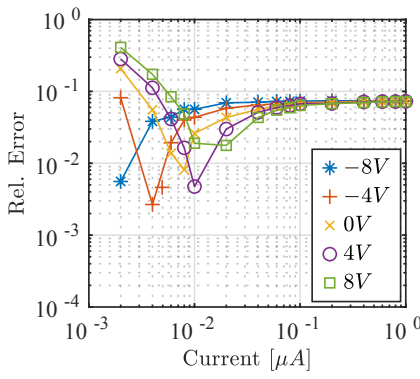

(b)

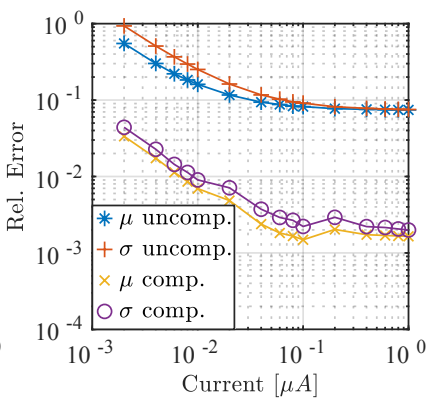

(d)
Fig. 5. Uncompensated relative current error and impact of the load voltage for a single channel for (a) a positive current and (b) a negative current. Compensated relative current error $(\mu$ and $\sigma$ ) for all load voltages for (c) a positive current and (d) a negative current.

relative current error ( $\mu$ and $\sigma$ ) for the full range of load voltages without and with realtime digital compensation (eq. 5). The relative error is at least $\mathrm{x} 10$ lower after compensation and independent of the load voltage. The current channel-tochannel mismatch is $<1.2 \%$ for $I \leq 10 \mathrm{nA}$ and $<0.1 \%$ for $I>10 \mathrm{nA}$.

Next, the voltage sensing accuracy is assessed. Because this block contains less circuitry, it matches more closely to the ADC limitations. The average relative voltage accuracy is $<10 \%$ for $V \leq 10 \mathrm{mV}$ and $<1 \%$ for $V>10 \mathrm{mV}$. The channel-to-channel mismatch is $<7 \%$ for $V \leq 10 \mathrm{mV}$ and $<1 \%$ for $V>10 \mathrm{mV}$.

\section{B. Electrochemical Verification}

Electrochemical experiments were done using a solution of potassium ferrocyanide $(5 \mathrm{mM}$ with $1 \mathrm{M} \mathrm{NaCl}$ to improve the conductivity) without mixing. Each working electrode measured $500 \times 500 \mu \mathrm{m}$ with a gold surface finish. The reference electrode was an $\mathrm{Ag}-\mathrm{AgCl}(3 \mathrm{M} \mathrm{KCl})$ with potential of $0.205 \mathrm{~V}$ v. the standard hydrogen electrode (ALS, Japan). The common counter electrode was a platinum wire. The parasitic channel capacitance current was measured and subtracted in postprocessing.

A CV has been performed on all channels simultaneously for a scan rate of $10 \mathrm{mV} \mathrm{s}^{-1}$ (Fig. 7a) and for a scan rate of $50 \mathrm{mV} \mathrm{s}^{-1}$ (Fig. 7b). The average current $\mu$ and the standard deviation $\sigma$ of the current of the second cycle are plotted. The

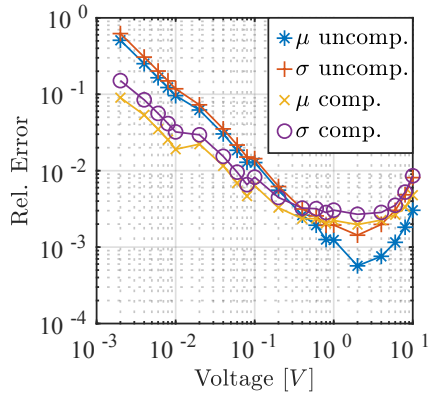

(a)

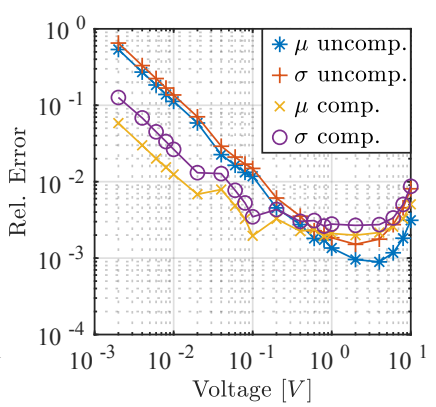

(b)
Fig. 6. Comparison between the relative voltage error ( $\mu$ and $\sigma$ ) for uncompensated and compensated channels for (a) negative voltages and (b) positive voltages.

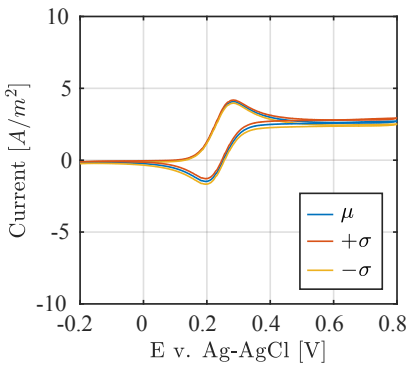

(a)

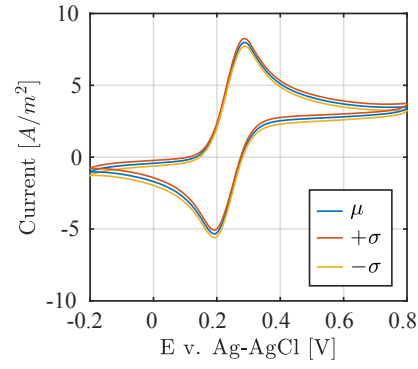

(b)
Fig. 7. Simultaneous cyclic voltammetry ( $\mu$ and $\sigma$ ) of ferrocyanide with a scan rate (a) of $10 \mathrm{mV} \mathrm{s}^{-1}$ and (b) of $50 \mathrm{mV} \mathrm{s}^{-1}$.

resulting $\mathrm{CV}$ corresponds to a typical $\mathrm{CV}$ for ferrocyanide with a midpoint potential independent of the scan rate and extrema currents dependent of the scan rate [10]. The majority of the observed variation can be attributed to the variation present in the electrodes.

Lastly, Fig. 8 shows the result of a simultaneous chronoamperometry experiment with a different step for each channel, from $0.1 \mathrm{~V}$ to $(-0.2+i / 128) \mathrm{V}$. This experiment illustrates the individual stimulation and sensing capabilities of the developed 128-channel potentiostat. As expected, a negative step results in a temporal negative current (to discharge the interface capacitance), while a positive step results in a positive current (to charge the interface capacitance). The larger the step, the larger the current spike, ultimately limited by the maximum current of the system [10]. Without parallelism, this experiment would take $128 \mathrm{x}$ longer ( $43 \mathrm{~min}$ instead of only $20 \mathrm{~s}$.

\section{Comparison with the SotA}

The performance of the 128-channel potentiostat is first compared with the multichannel SotA potentiostats using PCB technology (Table II). The system outclasses the SotA PCB systems on the amount of individual stimulation (20x) and sensing $(5 \mathrm{x})$ channels for only a fraction of the cost (4x lower) and area (5x smaller). Furthermore, the channel performance 
TABLE II

COMPARISON OF THIS WORK WITH THE SOTA

\begin{tabular}{l|c|c|c|c|c}
\hline \hline Instrument & $2012[5]$ & $2018[7]$ & $2019[4]$ & $2019[8]$ & This work \\
\hline Technology & $\mathrm{PCB}$ & $\mathrm{PCB}$ & $\mathrm{PCB}$ & $\mathrm{PCB}$ & $\mathbf{P C B}$ \\
Current range (resolution) & $\pm 10 \mu \mathrm{A}(5 \mathrm{pA})$ & - & $\pm 100 \mu \mathrm{A}(6 \mathrm{nA})$ & $\pm 4.9 \mathrm{~mA}(150 \mathrm{nA})$ & $\pm \mathbf{3 . 3} \boldsymbol{A}(\mathbf{1 0 0} \boldsymbol{p A})$ \\
Current accuracy (\# bits) & - & $-(20)$ & - & $0.1 \%(16)$ & $\mathbf{1} \%(\mathbf{1 6})$ \\
Voltage range (resolution) & $\pm 2 \mathrm{~V}(3 \mu \mathrm{V})$ & - & $\pm 1.2 \mathrm{~V}(600 \mu \mathrm{V})$ & $\pm 2.56 \mathrm{~V}(78 \mu \mathrm{V})$ & $\pm \mathbf{1 0} \boldsymbol{V}(\mathbf{3 0 5} \mu \boldsymbol{V})$ \\
Voltage accuracy (\# bits) & - & $-(24)$ & $2 \%(12)$ & $0.01 \%(16)$ & $\mathbf{1} \%(\mathbf{1 6})$ \\
Voltage ripple & none & - & none & none & $\approx \mathbf{2 0} \boldsymbol{m \boldsymbol { V }} \mathbf{1} \boldsymbol{\mathbf { A }}$ \\
Stimulation channels & 1 & 0 & 1 & 6 & $\mathbf{1 2 8}$ \\
Measurement channels & 24 & $6(V)+2(I)$ & 1 & 6 & $\mathbf{1 2 8}$ \\
Cost-per-channel & - & low-cost & $<20 \$$ & $<50 \$$ & $<\mathbf{5}$ \\
Area-per-channel & - & $\approx 1 \times 10^{3} \mathrm{~mm}^{2}$ & $\approx 540 \mathrm{~mm}^{2}$ & $\approx 530 \mathrm{~mm}^{2}$ & $\approx \mathbf{9 3} \mathbf{m m}^{\mathbf{2}}$ \\
\hline \hline
\end{tabular}

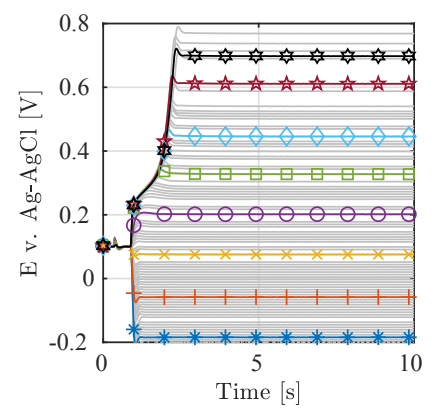

(a)

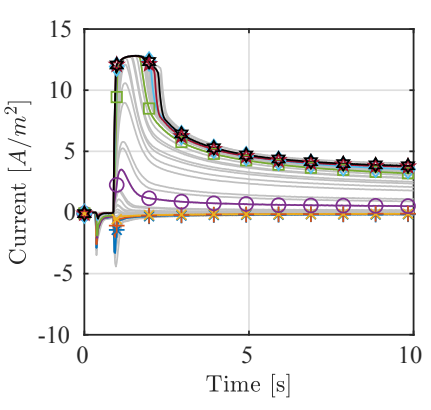

(b)
Fig. 8. Simultaneous chronoamperometry for all channels $(i=1 \ldots 128)$ from $0.1 \mathrm{~V}$ to $(-0.2+i / 128) \mathrm{V}$ with (a) the potential and (b) the current over time. Of all channels, plotted in grey, eight distinct ones, with uniform spacing, are colored and marked.

matches the SotA, except for the relative performance of [8] and the number of bits in [7], although no effective accuracy is reported. A small steady-state voltage ripple is present at the output. Future work should focus on a faster current pump to reduce this ripple below the detection limit.

Next, in comparison with advanced cmos technology, our system has $2 \mathrm{x}$ more parallel stimulation channels than [9]. However, up to 1024 parallel measurement channels (but with a single stimulation controller) have already been proposed [6]. Despite the major advantage of cmos technology in the required area-per-channel, the on-chip electrodes require a challenging post-processing procedure to make them biocompatible. Moreover, external electrodes are not supported, making the system less flexible in use.

\section{CONCLUSION}

This work presented a 128-channel potentiostat to speed up (bio)electrochemical experiments which are known to have a slow response, an environmental dependency and an inherent variation. The introduced multichannel potentiostat architecture makes extensive use of time-sharing to obtain 128 individual stimulation and sensing channels, 20x more than the SotA PCB systems. The system is implemented with PCB technology resulting in a cost-per-channel of $<5 \$, 4 \mathrm{x}$ lower than the SotA, and an area-per-channel of $\approx 93 \mathrm{~mm}^{2}, 5 \mathrm{x}$ lower than the SotA. Realtime digital compensation minimizes the channel-to-channel mismatch to $<1 \%$. Two electrochemical experiments on ferrocyanide were run: a CV in parallel on all channels and a chronoamperometry with 128 different steps illustrating a best-case speedup of $128 \mathrm{x}$.

\section{ACKNOWLEDGMENT}

The work of T.R. Molderez was supported by a Doctoral Fellowship of the Research Foundation Flanders (FWO).

\section{REFERENCES}

[1] B. E. Logan and K. Rabaey, "Conversion of wastes into bioelectricity and chemicals by using microbial electrochemical technologies," Science, vol. 337, no. 6095, pp. 686-690, 2012.

[2] X. Zhang, A. Prévoteau, R. O. Louro, C. M. Paquete, and K. Rabaey, "Periodic polarization of electroactive biofilms increases current density and charge carriers concentration while modifying biofilm structure," Biosensors and Bioelectronics, vol. 121, pp. 183 - 191, 2018.

[3] V. Bianchi, A. Boni, S. Fortunati, M. Giannetto, M. Careri, and I. De Munari, "A wi-fi cloud-based portable potentiostat for electrochemical biosensors," IEEE Transactions on Instrumentation and Measurement, pp. 1-8, 2019.

[4] S. D. Adams, E. H. Doeven, K. Quayle, and A. Z. Kouzani, "Ministat: Development and evaluation of a mini-potentiostat for electrochemical measurements," IEEE Access, vol. 7, pp. 31 903-31 912, 2019.

[5] M. Vergani, M. Carminati, G. Ferrari, E. Landini, C. Caviglia, A. Heiskanen, C. Comminges, K. Zor, D. Sabourin, M. Dufva, M. Dimaki, R. Raiteri, U. Wollenberger, J. Emneus, and M. Sampietro, "Multichannel bipotentiostat integrated with a microfluidic platform for electrochemical real-time monitoring of cell cultures," IEEE Transactions on Biomedical Circuits and Systems, vol. 6, no. 5, pp. 498-507, 2012.

[6] A. Manickam, K. You, N. Wood, L. Pei, Y. Liu, R. Singh, N. Gamini, D. Shahrjerdi, R. G. Kuimelis, and A. Hassibi, "11.2 a cmos biosensor array with 10243 -electrode voltammetry pixels and 93db dynamic range," in 2019 IEEE International Solid- State Circuits Conference (ISSCC), 2019, pp. 192-194.

[7] G. C. Zafeiropoulos, K. I. Papadimitriou, and E. M. Drakakis, "Performance and accuracy in electrical bioactivity recordings (panacea): A high-performance, wireless, multi-instrument for potentiometric and amperometric recording of biosignals," Measurement, vol. 129, pp. 128 - 141, 2018.

[8] T. R. Molderez, X. Zhang, K. Rabaey, and M. Verhelst, "A currentdriven six-channel potentiostat for rapid performance characterization of microbial electrolysis cells," IEEE Transactions on Instrumentation and Measurement, pp. 1-9, 2019.

[9] P. Li, T. R. Molderez, F. Ceyssens, K. Rabaey, and M. Verhelst, "A 64channel, 1.1-pa-accurate on-chip potentiostat for parallel electrochemical monitoring," in 2019 IEEE 45th European Solid-State Circuits Conference (ESSCIRC), September 2019.

[10] A. J. Bard and L. R. Faulkner, Electrochemical Methods: Fundamentals and Applications, 2nd ed. Wiley, 2000. 